Diderik Batens. Adaptive logics as a necessary tool for relative rationality. Including a section on logical pluralism. In Erik Weber, Dietlinde Wouters, and Joke Meheus, editors, Logic, Reasoning and Rationality, pages 1-25. Springer, Dordrecht, 2014 


\title{
Adaptive Logics as a Necessary Tool for Relative Rationality. Including a Section on Logical Pluralism
}

\author{
Diderik Batens
}

\section{Aim of this Paper}

In most papers on adaptive logics, for example [9, 12], and in the forthcoming book [14], I try to remain philosophically neutral on whatever is not strictly relevant for adaptive logics. People with different political viewpoints may play the violin, or handle a hammer. Similarly, people with different philosophical viewpoints may apply the same adaptive logics, which may be sensibly classified as reasoning instruments. Tying those logics to my specific philosophical convictions would scare away some readers.

Of course, I have philosophical convictions. Especially those in the realm of epistemology motivated the origin and especially the development of the adaptive logic program. To make this link explicit seems useful. Adaptive logics clarify the notion of defeasible reasoning and highlight its importance. By doing so, they evoke a number of epistemological questions and rule out certain epistemological answers to these questions. The questions are far from specific for my epistemological views, but present interesting problems for any epistemological view.

So the potential interest of the present paper is double. Adaptive logics were developed in view of a philosophical need, which is to make a certain epistemological position meaningful and precise. These logics represent precise formulations of methods and actually of a multiplicity of alternative methods-plurality is easy from an adaptive perspective. The resulting problem for any epistemological stand is: In which way may such methods be integrated?

I shall begin by a, necessarily rough, sketch of my epistemological stand. This is meant as a point of reference and as an example of a stand in which defeasible reasoning, especially as approached by adaptive logics, finds its natural place.

Diderik Batens

Centre for Logic and Philosophy of Science, Universiteit Gent, Belgium, e-mail: Diderik.Batens@UGent.be 
After a quick rehearsal of adaptive logics, formal problem-solving processes are introduced. In these, adaptive logics have their natural place and are able to show their strength. One or more adaptive logics are here combined with a deductive logic as well as with an erotetic logic.

In the next four sections, some typical features will be outlined. I chose those that are presumably most controversial and at the same time most difficult to incorporate within a formal framework. (i) Contextual meaning of logical terms is handled within a formal framework rather than within a linguistic one. The discussion concerns the way in which formal properties of the premises determine the meaning of occurrences of logical terms. (ii) The section on the meaning of logical symbols concerns the distinction that some want to draw between deductive and defeasible reasoning forms and the effects of this distinction on the meaning of logical terms. (iii) The next topic is the contextual meaning of non-logical terms. (iv) The traditional notion of a theory is confronted with an alternative in the section on complex theories. The central issue is that traditional theories may fail to be efficient means to embody the best available human knowledge. While complex theories have the disadvantages of their complexity, including their computational complexity, they are able to describe complex domains that are beyond the reach of traditional theories. (v) The section on logical pluralism is mainly meant to clarify a form of logical pluralism that does not coincide with most positions defended and attacked in the literature.

\section{Epistemological Stand}

This very compressed sketch will consist of a set of theses, each followed by one or more arguments. An extensive description of my epistemological stand is unfortunately only available in [4] (and in its Greek translation). Some aspects are also discussed in $[1,2,3,5,20]$.

The first thesis reads: All knowledge is ultimately defeasible. Note that it says that all knowledge is defeasible, not that all reasoning is defeasible. Still, even nondefeasible reasoning starts always from defeasible premises, whence its conclusions are also defeasible. As we shall see, not all knowledge is defeasible in the same sense or in the same way.

Many will agree that most empirical knowledge is defeasible. Inductive generalizations clearly are, and so are predictions. Most results of abductive reasoning, which includes explanations, are also defeasible. The same holds for knowledge of causal relations, expectancies, results of diagnostic reasoning, and so on. While all this clearly holds for knowledge about the physical world, knowledge about other humans is no exception. Statements made by trustworthy human beings need to be interpreted, for example by means of Gricean maxims, which introduce additional defeasibility - I write "additional" because the statements already rely on defeasible knowledge of the person who utters the statement. 
Let us turn to experience. That experience is never uninterpreted seems generally accepted today. I do not know any serious philosopher who identifies the set of experiential data with Mach's Empfindungen. Especially the philosophy of science of the second half of the twentieth century clarified this matter. Many reject the consequences attached to the insights of that period, especially the different forms of incommensurability. Yet nobody adduced any good arguments for questioning the insights themselves, which concern theory-ladenness. So even when we may have no reasons to mistrust our eyes or other senses, we may be interpreting what we see, etc., in a mistaken framework. So much more important than occasional optical or other illusions is the fact that every experience is interpreted and that this interpretation may be very mistaken, as the history of the sciences readily reveals.

The defeasible character of experience is enhanced by the fact that most so-called experience is the result of abduction. We think to see that it has rained because we think to see that the grass is wet, and we think to see that it has snowed because we think to see that our environment is covered with white stuff. We think to see a magpie because what we 'see' is compatible with what we know about magpies and not with what we know about any other species of birds. Such abductions occur usually in an unconscious way. Note that we are on a slippery slope here. There is a smooth transition from unconscious abduction to theory-laden observation.

Some will argue that we have means to obtain a higher certainty on our observations: repetition, instruments, experience, and so on-I return to these soon. However, the very fact that observations may be corrected and that means to obtain more reliable observations have been devised highlights the defeasibility of experience.

Methodological knowledge (norms and values in general) have long been claimed to be a priori. Today, informed people have changed their mind basically because history teaches us that methods are historically contingent and hence clearly defeasible. In trying to gather knowledge about the world, we learn how to learn. As Dudley Shapere candidly puts it: "what better basis could we have than what we have learned, including what we have learned about how to learn" [23, p. 52]. Shapere couples this with the idea that science is content-guided. He opposes this idea to the views of the Vienna Circle-its members soon turned to $\operatorname{logic}^{1}$ - as well as to the views of the 'post-classical' philosophers of science (Hanson, Kuhn, Toulmin, and Feyerabend) - these fell into extreme relativism.

Finally, I come to the most touchy and controversial point, logical and mathematical knowledge, even if I do not understand that any sane person could hold such knowledge to be non-defeasible. The point is not whether, for example, $0^{\prime}+0^{\prime}=0^{\prime \prime}$ is a theorem of Peano Arithmetic. The point is whether Peano Arithmetic is complete, non-trivial, suitable (in the sense in which Euclidian Geometry is not suitable to describe our universe), and so on. The point is also whether we have the right view on what it means to be a theorem of Peano Arithmetic. We know from Gödel's First Incompleteness Theorem that Peano Arithmetic is incomplete if it is consistent. Whether it is non-trivial we do not know. Most mathematicians think it is, but

\footnotetext{
${ }^{1}$ This paper relies on a conception of logic, and even of formal logic, which is different from the Vienna Circle's conception and leaves ample room for content-guidance-see [11] for an elaboration.
} 
Graham Priest has argued in [21] that it is not, at least not if it is extended with some obviously correct proof means that do not undermine its semi-recursive character. Moreover, we know from Gödel's Second Incompleteness Theorem that, if Peano Arithmetic is non-trivial, it is impossible to show so by means that can be represented within Peano Arithmetic. ${ }^{2}$ Note that, if Peano Arithmetic is trivial, the statement that $0^{\prime}+0^{\prime}=0^{\prime \prime}$ is one of its theorems obtains a rather unexpected meaning. If Priest is right, the whole realm of mathematics has to be rethought, presumably starting from an inconsistent set-theory and working our way down to mathematical theories that we apply in everyday life.

We have known more revolutions in the history of mathematics. Many theories originated in a definite period. Their early history was often messy and full of nonsense. The early history of algebra is a ready example. Many years were required before one arrived at symbolic algebra and only then was sense made of isolated negative terms - see for example $[17,18]$. Some theories, like Newton's infinitesimal calculus or Cantor's set theory or Frege's set theory were later found to be mistaken, even nonsensical if taken literally. There are the other limitative theorems, all discovered less than a century ago. All this drastically changed the conception of mathematical theories.

So mathematical knowledge is at least defeasible in the sense that one's best insights at some point in time may be later superseded by insights that derive from further study, or from improved conceptual insights, or presumably also from the development of empirical sciences. I shall argue in Section 8 that the same holds for logic.

The second thesis is a consequence of the preceding one: No foundation is available in any domain. This means that the only way to arrive at justified convictions is to improve our knowledge by relying on our present knowledge. Of course, we may and should learn from the history of knowledge, especially in methodological respects. But even historiography is the result of our present insights. Obviously, we should collect new data. Still, which data have to be collected and the importance and significance that will be attached to them will depend on present lights. This is what I call relative rationality (and relative justification).

Two other theses are directly connected to the previous one, but space does not permit to explain the connection. The first thesis: All meaning is contextual. The (intensional) meaning of words does not reside in some Platonic heaven, but in people's heads. As such, they depend on one's view on the domain. Studying any outdated theory from the history of the sciences is convincing in this respect. The second thesis: Actual knowledge systems are neither holistic nor hierarchical. They consist of set of (larger or smaller, more or less vague) clusters of knowledge (about the world, methods, language, ....). These clusters are invoked to solve specific problems. They may be mutually inconsistent. Some of the clusters are related. (i) Some clusters are extended with consequences of others if the need presents itself-as when the knowledge related to handling everyday objects is extended with some physics or geometry to solve a specific problem. (ii) Some of the clusters are as-

${ }^{2}$ Even this Theorem is defeasible. It is proved by means that are only reliable if Peano Arithmetic is non-trivial. 
sociated with others-a set of methodological do's and don'ts may be related to a scientific discipline.

According to this viewpoint, the central epistemological tasks are the following. First of all, one has to solve problems at all 'levels', including the conceptual organization of scientific theories and their unification. Such tasks are subordinate to the central problems that have to be solved. Next, one often has to analyse the context (problem-solving situation) in order to reach a solution. A third and central task is to reduce the role of 'pragmatic factors', factors that depend on properties of the knowing person or group rather than on the domain studied. Note that this reduction takes always place on the basis of available knowledge; it is not a matter of reaching more 'objectivity'. Another central task is furthering intellectual combat. Intellectual fight prevailed in all pivotal periods of the history of the sciences. It is the only means to discover the weak spots in our convictions and to strengthen them.

Subsequent sections are intended as an answer to the question whether the outlined epistemological stand makes sense from a logical point of view and whether it can be backed up by logical means.

\section{Adaptive Logics: A Quick Rehearsal}

An adaptive logic is a formal logic that 'adapts itself to the premises' and characterizes a defeasible reasoning form. The ultimate aim of the adaptive logic program is to characterize all defeasible reasoning forms by an adaptive logic in standard format.

In standard format, an adaptive logic is defined by a triple: (i) a lower limit logic LLL, roughly a deductive Tarski logic that is compact and for which there is a positive test, ${ }^{3}$ (ii) a set of abnormalities $\Omega$, which is characterized by a (possibly restricted) logical form, ${ }^{4}$ and (iii) an adaptive strategy: Reliability or Minimal Abnormality. ${ }^{5}$

The standard format provides the adaptive logic with dynamic proofs. Typical for the annotated proofs is that each line has a possibly empty condition, which is a finite set of abnormalities. The lower limit logic and the set of abnormalities determine the inferential rules (in terms of LLL-consequence), the set of abnormalities and the strategy determine the Marking definition. This definition proceeds in terms of the formulas that occur at the stage of the proof. Marked lines are considered OUT — their formula is not derived at the stage — while unmarked lines are

\footnotetext{
${ }^{3}$ There is a positive test for a logic $\mathbf{L}$ iff $\left\{\langle\Gamma, A\rangle \mid \Gamma \vdash_{\mathbf{L}} A ; A\right.$ is a formula; $\Gamma$ is a recursive set of formulas $\}$ is a semi-recursive set. A more general description is that $\mathbf{L L L}$ is any logic that has static proofs, but space prevents me from clarifying this here.

${ }^{4}$ For example, a formula of the form $\exists(A \wedge \neg A)$ or a formula of the form $\exists A \wedge \exists \neg A$, in which $\exists A$ denotes the existential closure of $A$. A possible restriction is that $A$ is a primitive (or atomic) formula, or that $A$ is a disjunction of primitive formulas and negations of primitive formulas.

5 These two strategies handle derivable disjunctions of abnormalities in different ways and have a different effect on the proofs and on the selection semantics.
} 
considered IN. As the proof proceeds from one stage to the next, a marked line may become unmarked and vice versa. Note that the marks are a means to control the defeasible character of the logic: a formula is derivable or underivable from the premises in view of the insights in the premises that is offered by the stage of the proof. A definition settles which formulas are finally derivable from the premises. As (full-blown) defeasible logics have no positive test for final derivability, a finite proof in itself will often not enable one to decide that a formula is finally derivable from the premises; one needs a metatheoretic reasoning about possible extensions of the proof.

The standard format also provides adaptive logics with a semantics, viz. a selection semantics. The lower limit logic assigns a set $S$ of models to the premise set; the set of abnormalities and strategy select a subset of $S$ as the set of adaptive models of the premises.

Finally, the standard format also provides most of the metatheory: Soundness and Completeness of the proof theory with respect to the semantics, and a host of further metatheoretic properties.

Incidentally, the logic obtained by extending LLL with an axiom that connects abnormalities to triviality (or by weeding out models that verify an abnormality) is called the upper limit logic ULL. A remarkable property of adaptive logics is that $C n_{\mathrm{AL}}(\Gamma)=C n_{\mathrm{ULL}}(\Gamma)$ whenever $\Gamma$ is normal (does not require any abnormalities to be true), whereas $C n_{\mathbf{L L L}}(\Gamma) \subseteq C n_{\mathbf{A L}}(\Gamma) \subset C n_{\mathbf{U L L}}(\Gamma)=\mathscr{W}$ (with $\mathscr{W}$ the set of all formulas) whenever $\Gamma$ is abnormal.

Adaptive logics are called corrective iff they handle premise sets that have no models in the 'standard logic'; otherwise they are called ampliative. Where $\mathbf{C L}$ (classical logic) is taken to be the standard, adaptive logics handling inconsistencies are corrective, while adaptive logics for inductive generalization are ampliative. For a more detailed description of the distinction, I refer for example to $[9,12,14]$.

\section{Formal Problem-Solving Processes}

Logics are not applied in isolation. This holds especially when adaptive logics are involved. Such logics are precise characterizations of methods and are at least combined with a deductive logic, usually their own lower limit logic. Obviously, several adaptive logics may be combined. This is the case, for example, when we look for an explanation of a fact in terms of present knowledge, but try to extend our knowledge with new inductive generalizations ${ }^{6}$ in case our present knowledge does not provide an explanation.

Reasoning is a goal-directed and problem-oriented process. So we need to be able to express problems and the process should be sensible in view of the solution of the problem or problems. Moreover, we need to be able to ascertain whether a

\footnotetext{
${ }^{6}$ Many logicians appear to be mesmerized by the grue paradox. However, this concerns the choice of a language (or of a set of primitive predicates), which has obviously to be justified by non-logical means: entrenchment, etc.
} 
problem is well-formed in view of the available declarative knowledge, we need to be able to split up problems, and to derive problems from given problems in view of declarative knowledge. More often than not, the solution of a problem requires empirical import. This is obviously not provided by any logic, but the adaptive logic should trigger the empirical import; it should instruct one whether new empirical data may be relevant or not.

The required combination is realized by means of formal problem-solving processes, fpsps for short. These were first proposed in [8]. In [11] it was shown that fpsps leave ample room for, and actually install, content-guidance; it was also shown that building in observational or experimental means requires an 'oracle' that is different from the one introduced by Hintikka in [19]. So let me describe the elements of a fpsp backbone.

An fpsp is a sequence of lines that results from a procedure. This is a set of instructions that consist of a rule with a permission or obligation attached to it; the permission or obligation should be defined in terms of the lines that already occur in the fpsp. A fpsp contains two kinds of lines.

Declarative lines are quadruples: a line number, a prospective expression of the form $\left[B_{1}, \ldots, B_{n}\right] A$, a justification, and an adaptive condition. The prospective expression states that $A$ can be obtained from the premises iff the members of the prospective condition, $\left[B_{1}, \ldots, B_{n}\right](n \geq 0)$, can be obtained from them. The prospective dynamics is basically a way to push part of the proof heuristics into the proof (see [16]); the members of the prospective condition function as targets in view of which premises are introduced and analysed. Moreover, the prospective dynamics leads to criteria for final derivability in adaptive logics (see $[10,26])$. The adaptive conditions are those of the involved adaptive logics; they are essential for the marking and hence for the control of the involved defeasible reasoning forms-see Sect. 3 .

Problem lines are couples: a problem, which is phrased as a set of yes-no questions $\left\{?\left\{A_{1}, \neg A_{1}\right\}, \ldots, ?\left\{A_{n}, \neg A_{n}\right\}\right\}$ and a justification. Problems are handled in terms of Andrzej Wiśniewski's erotetic logic (see [30, 31, 29, 28]), which does not presuppose a specific deductive logic but is defined in a general way. The erotetic logic handles the evocation of questions by declarative premises and the implication of questions by other questions in view of declarative premises. As fpsps (by present lights) start from a main problem and a set of premises, only question implication plays a role. Note that deriving a sub-problem from a problem is a logical matter, whereas deriving an 'auxiliary' problem requires declarative knowledge.

Apart from Wiśniewski's erotetic logic, which handles questions, there are specific rules to handle problems. Thus, once an answer to ? $\left\{A_{i}, \neg A_{i}\right\}(1 \leq i \leq n)$ is obtained - once $A_{i}$ or $\neg A_{i}$ is derived on an empty prospective condition- the problem $\left\{?\left\{A_{1}, \neg A_{1}\right\}, \ldots, ?\left\{A_{n}, \neg A_{n}\right\}\right\}-\left\{?\left\{A_{i}, \neg A_{i}\right\}\right\}$ is derivable from the problem $\left\{?\left\{A_{1}, \neg A_{1}\right\}, \ldots, ?\left\{A_{n}, \neg A_{n}\right\}\right\}$. As soon as the former is derived, the latter becomes redundant.

The main problem is essential for the goal-directed character of fpsps. A prospective proof normally starts with a (redundant) goal statement of the form $[A] A$, which is meant to introduce $A$ as a target. In an fpsp, goal statements are derived from 
non-redundant problems. This means that $[A] A$ can only be introduced if ? $\{A, \neg A\}$ is a member of a non-redundant problem. Once targets are present, the prospective dynamics warrants the goal-directed character of the fpsp; a new line can only be added if its prospective expression potentially brings us closer to deriving a target. Whether it actually brings us closer to deriving a target depends on the premises and cannot in general be settled beforehand, given that we are not logically omniscient. Finally, new problems are only introduced if derived declarative statements warrant that their solution is useful for solving given problems in view of the declarative premises.

Note that fpsps guides research. The introduction of a goal statement $[A] A$ will, after a number of steps, lead to the presence of expressions of the form $\left[B_{1}, \ldots, B_{n}\right] A$. This suggests observations, experiments, conceptual analysis, or bringing in other information, and all of these will lead to new premises. New premises will be re-

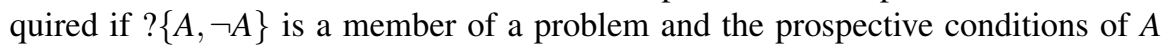
and $\neg A$ cannot be derived from the premises. Even if the question can be answered by deductive means, a new premise may be more easily obtainable by observation than by deduction.

I only presented an outline of the backbone of fpsps. Still, having referred the reader to other papers, I should stress that lots of work still has to be carried out. Thus the incorporation of some adaptive logics in fpsps requires that the relevant heuristics is elaborated. All this, however, is pretty standard or at least does not require much ingenuity.

\section{Contextual Meaning}

The first point I shall make is that adaptive logics introduce contextual meanings. To see this, consider a simple propositional example of a proof for the logic $\mathbf{C L u N}{ }^{r}$. Its lower limit logic (generic name: $\mathbf{L L L}$ ) is $\mathbf{C L u N}$, which is full positive propositional logic together with excluded middle. The set of propositional abnormalities comprises the formulas of the form $A \wedge \neg A$-the predicative abnormalities are the existential closure of those formulas, in which $A$ is then possibly open. The strategy is Reliability—see below.

The (generic) rules of inference are the same for all (non-combined) adaptive logics. Let

\section{$A \quad \Delta$}

abbreviate that $A$ occurs in the proof on the condition $\Delta$, which is a set of abnormalities (so a subset of $\Omega$ ). There are three generic rules. In RC, $\breve{V} \Theta$ denotes the classical disjunction of the members of $\Theta \subset \Omega$ and the symbol $\breve{V}$ is the classical disjunction. ${ }^{7}$

\footnotetext{
${ }^{7}$ I skip some related complications. They are not relevant to the point I am trying to make here.
} 
Prem If $A \in \Gamma$ :

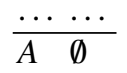

RU

$$
\text { If } A_{1}, \ldots, A_{n} \vdash_{\mathbf{L L L}} B: \quad \begin{array}{ll} 
& A_{1} \Delta_{1} \\
& \ldots \ldots \\
& \frac{A_{n} \Delta_{n}}{B \quad \Delta_{1} \cup \ldots \cup \Delta_{n}}
\end{array}
$$

\begin{tabular}{|c|c|c|c|}
\hline 1 & $(\neg p \wedge \neg q) \wedge t$ & PREM & $\emptyset$ \\
\hline 2 & $p \vee r$ & PREM & $\emptyset$ \\
\hline 3 & $q \vee s$ & PREM & $\emptyset$ \\
\hline 4 & $p \vee q$ & PREM & $\emptyset$ \\
\hline 5 & $t \supset p$ & PREM & $\emptyset$ \\
\hline 6 & $r$ & 1,$2 ; \mathrm{RC}$ & $\{p \wedge \neg p\}$ \\
\hline 7 & $s$ & 1,$3 ; \mathrm{RC}$ & $\{q \wedge \neg q\}$ \\
\hline 8 & $(p \wedge \neg p) \vee(q \wedge \neg q)$ & 1, 4; RU & $\emptyset$ \\
\hline
\end{tabular}

$$
\begin{array}{ll}
\mathrm{RC} \quad \text { If } A_{1}, \ldots, A_{n} \vdash_{\mathbf{L L L}} B \grave{\vee} \boldsymbol{V} \Theta: & A_{1} \Delta_{1} \\
& \ldots \ldots \\
& \frac{A_{n} \Delta_{n}}{B \quad \Delta_{1} \cup \ldots \cup \Delta_{n} \cup \Theta}
\end{array}
$$

Let the premise set be $\{(\neg p \wedge \neg q) \wedge t, p \vee r, q \vee s, p \vee q, t \supset p\}$. Here is the proof up to stage $8:^{8}$

Lines 6 and 7 are marked at this stage of the proof. Where Reliability is the strategy, a line is marked at a stage iff its condition contains a disjunct of a minimal disjunction of abnormalities. At stage 8 of the proof, the only minimal disjunction of abnormalities is the formula of line 8 . Let us now consider stage 9 of the proof-I rewrite the sequence of lines from line 6 on.

$\begin{array}{lllll}6 & r & 1,2 ; \mathrm{RC} & \{p \wedge \neg p\} & \sqrt{ } \\ 7 & s & 1,3 ; \mathrm{RC} & \{q \wedge \neg q\} & \\ 8 & (p \wedge \neg p) \vee(q \wedge \neg q) & 1,4 ; \mathrm{RU} & \emptyset & \\ 9 & p \wedge \neg p & 1,5 ; \mathrm{RU} & \emptyset\end{array}$

Line 6 is still marked, but line 7 is unmarked. Indeed, the only minimal disjunction of abnormalities at stage 9 is the formula of line 9 , viz. $p \wedge \neg p .{ }^{9}$

In all subsequent stages of the proof-in more traditional terms: in all extensions of this proof-line 6 is marked and that line 7 unmarked. In other words, the proof at stage 9 is stable with respect to lines 6 and 7 (and actually with respect to lines $1-7)$. So $s$ is finally derivable from $\Gamma$, whereas $r$ is not. ${ }^{10}$

\footnotetext{
${ }^{8}$ A stage is a sequence of lines and a proof is a chain of stages.

${ }^{9}$ The Minimal Abnormality strategy leads, for some premise sets, to a richer consequence set than the Reliability strategy. For this proof, however, both strategies lead to the same marks.

${ }^{10}$ The definition of final derivability is slightly more sophisticated, but this is what it comes to for the present propositional example.
} 
The above proof nicely illustrates the contextual meaning of negation. The negation in $\neg p$ is clearly the paraconsistent CLuN-negation because both $p$ and $\neg p$ are $\mathbf{C L u N}^{r}$-derivable from the premises. The negation in $\neg q$ has the force of a CLnegation. Precisely this is why $s$ is a consequence of the premises: $s$ follows from $\neg q$ and $q \vee s$ because the premises do not require that $q \wedge \neg q$ is a disjunct of a true and minimal disjunction of abnormalities. ${ }^{11}$

To understand what is going on, let us have a look at the preferred application context of inconsistency-adaptive logics. Consider a theory $T$, that is intended as consistent and has $\mathbf{C L}$ as its underlying logic, but turns out to be inconsistent. Often, one will try to find a consistent replacement $T^{\prime}$, and one will try to obtain it by reasoning from $T$. One typically will want a $T^{\prime}$ that is as rich as $T$, except that $T^{\prime}$ should be consistent. While $\mathbf{C L}$ is obviously useless for this purpose (it identifies $T$ with the trivial theory), (static) paraconsistent logics are too weak; many 'good' consequences of $T$ will not be derivable by the paraconsistent logic because it is much weaker than $\mathbf{C L}$.

Inconsistency-adaptive logics are obviously not intended to remove the inconsistencies. This should be done on the basis of non-logical arguments: new empirical data or new results of conceptual analysis. Incidentally, it is not difficult to devise adaptive logics that remove inconsistencies. However, some such logics lead to arbitrary results (removing, for example, $p$ in favour of $\neg p$ ) and some leave one with too poor a theory (when it removes both 'halves' of every inconsistency).

In preparation of removing the inconsistencies by non-logical means, we need to obtain a maximally consistent interpretation of $T$, from which the inconsistencies may then be removed. Inconsistency-adaptive logics should provide us with such an interpretation. The example proof illustrates the way in which they do so. Inconsistency-adaptive logics, like $\mathbf{C L u N}^{r}$, interpret premise sets as consistently as possible. ${ }^{12}$ In other words, inconsistencies are considered as false, except when the premises prevent this; for Reliability this comes to: except when the inconsistency is a disjunct of a minimal disjunction of abnormalities that is derivable from the premises by the lower limit logic. Precisely because inconsistency-adaptive logics interpret premise sets as consistently as possible, they assign a contextual meaning to negation. It is worth noting that the meaning that is assigned to a negation depends on the content of the premise set.

Incidentally, why do we need a maximal consistent interpretation of the theory? The theory $T$ was intended as closed under $\mathbf{C L}$ : every $\mathbf{C L}$-consequence of theorems of $T$ is itself a theorem of $T$. This causes $T$ to be trivial. We cannot look for a consistent replacement of $T$ itself, but we can look for a consistent replacement of the non-trivial theory that it closest to the original intention, and this is a maximal consistent interpretation of $T$. In it the inconsistencies are localized and all 'parts' in which no inconsistency is involved are closed under $\mathbf{C L} .^{13}$

\footnotetext{
${ }^{11}$ If the negation is paraconsistent and $A$ as well as $\neg A$ are true, then $A \vee B$ and $\neg A$ are true even if $B$ is false.

12 This expression is ambiguous and is disambiguated by the strategy.

${ }^{13}$ A maximal consistent interpretation can only be defined by a defeasible inference relation and the adaptive logic program aims at characterizing all such relations.
} 
There is a variety of inconsistency-adaptive logics. Each of them offers a variety of maximally consistent interpretations of $T$. In doing so, each of them assigns, for every formula of the form $\neg A$, one of (at least) two meanings to the $\neg$ in view of the premise set. These are the meanings of negation as fixed by respectively the lower limit logic and the upper limit logic. By varying the lower limit logic and the set of abnormalities - the latter may have effects on the upper limit logic-one varies the couples of negations that is chosen from. By varying the strategy and by certain variations of the set of abnormalities,${ }^{14}$ one may vary the choices made between a same couple of negations. A similar (but different) variation is obtained by combined adaptive logics. ${ }^{15}$ Incidentally, some combined inconsistency-adaptive logics assign contextual meanings to negations by choosing from more than two negations.

Do not complain about the large variety of possibilities. As was mentioned in Sect. 1, adaptive logics form precise formulations of methods, in the present case methods for handling inconsistency. The choice between such methods requires a philosophical justification, which should be contextual in that it should depend on the properties of the specific situation. More variation is spelled out in [14, Chap. 7].

There is, however, a very different multiplicity of adaptive logics for handling inconsistency. An inconsistency, viz. that some $A$ is true together with $\neg A$, may be seen as a negation glut. That $A$ as well as $\neg A$ are false may be seen as a negation gap. In a similar way, all logical terms (including the quantifiers and identity) may be said to display gluts or gaps (or both). One way to define the gluts and gaps is with respect to the $\mathbf{C L}$-truth conditions. For example, there is an implication glut if $A \supset B$ is true while $A$ is true and $B$ is false, and there is an implication gap if $A \supset B$ is false while $A$ is false or $B$ is true. The interesting point is that many inconsistent theories have models in which no negation gluts but other gluts or gaps occur. Whenever this is the case, the adaptive approach (which 'minimizes' abnormalities) leads to 'interpretations' of the inconsistent theory that are as normal as possible. This was explained already in [6] and the matter is studied at some length in [14, Chap. 8]. There I also consider the ambiguity of non-logical terms ${ }^{16}$ as well as its combination with kinds of gluts and gaps for logical terms. The combination of ambiguity with all kinds of gluts and gaps leads to zero logic, by which nothing is derivable from any premise set, not even the premises themselves. All the (Tarski) logics and combinations of them lead to adaptive logics that define a maximally normal interpretation of the premises. The adaptive logic that has zero logic as its lower limit may not be very interesting in itself, but it is an excellent instrument for

\footnotetext{
${ }^{14}$ Given a lower limit logic, there are often several sets of abnormalities that lead to the same upper limit logic.

${ }^{15}$ Several combined adaptive logics have been described. The simplest combination, which obviously works only under certain conditions, is where a consequence set of $\Gamma$ is defined as the union of the consequence sets different simple adaptive logics assign to $\Gamma$.

${ }^{16}$ Present ambiguity-adaptive logics do not assign any specific meanings to occurrences of nonlogical terms. They merely minimize the number of occurrences of the same term that require a different meaning.
} 
surveying which choices (of gluts, gaps and ambiguities) are sufficient to obtain a minimally abnormal interpretation of the premises.

So each of these adaptive logics offers a minimally abnormal interpretation of premise sets and introduces contextual meanings for all the logical terms for which they tolerate gluts or gaps. The same holds for ampliative adaptive logics-the matter is just a trifle more complicated.

\section{Meaning of Logical Symbols}

According to the official doctrine, a logic determines the meaning of its logical terms. By "logic" is meant a deductive logic here. What comes of this if adaptive logics are applied? Three positions seem sensible: the two-logic view, the dialetheist view, and the direct view.

According to the two-logic view, the meaning of logical terms is defined by the lower limit logic and the upper limit logic, which may be seen as deductive logics. The adaptive logic picks the right choice for each occurrence of the logical term. It picks the meaning from the upper limit logic whenever this is possible-a matter disambiguated and determined by the strategy.

If the adaptive logic $\mathbf{A L}$ is corrective, it offers a minimally abnormal interpretation of a theory or premise set. If the theory is normal, $\mathbf{A} \mathbf{L}$ offers a normal interpretation-say the $\mathbf{C L}$-interpretation. If the theory is abnormal, $\mathbf{A L}$ offers an interpretation according to which some logical terms have a meaning that is weaker than the CL-meaning. In the preferred application context, the result will eventually be replaced, on non-logical grounds, by a normal theory. So the contextual meanings are provisional; they apply in a transitory period in which problems have still to be solved in order to reach the 'finished' and normal theory.

It is worth noting that even the transitory stage ${ }^{17}$ can be made fully transparent from a logical point of view. Suppose for example that we are dealing with a simple adaptive logic which has $\mathbf{C L}$ as its upper limit and some weaker logic LLL as its lower limit. Let the standard logical symbols be those of LLL. It is obviously possible to enrich the language of $\mathbf{L L L}$ with a set of logical symbols that have the same meanings as the symbols of $\mathbf{C L}$ - the Ghent standard is to use 'checked' symbols

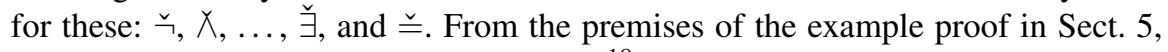

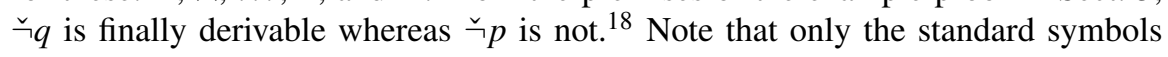
occur in the premises and that the adaptive logic determines which negations may be replaced by the $\mathbf{C L}$-negation.

As announced in Sect. 5, the matter is slightly more complicated for ampliative adaptive logics (such as adaptive logics for inductive generalization, for abduction, and so on). These adaptive logics offer a richer consequence set than $\mathbf{C L}$ (which I

\footnotetext{
${ }^{17}$ See the previous paragraph: the stage at which the adaptive theory is not yet replaced by a novel normal theory.

${ }^{18}$ Except for negation, all logical symbols of CLuN have the same meaning as the classical logical symbols.
} 
here consider as the standard of deduction for merely pragmatic reasons). To take a concrete example, consider inductive generalization and let the set of abnormalities be $\exists A \wedge \exists \neg A$ in which $\exists A$ is the existential closure of $A .{ }^{19}$ The upper limit logic is the so-called uniform classical logic (it has $\exists A \supset \forall A$ as a theorem and, in its models, the assignment value of every unary predicate is either the empty set or the whole domain; and similarly for other predicates). A typical application of the conditional rule, $\mathrm{RC}$, is that from $P a$ on the empty condition follows $\forall x P x$ on the condition $\exists x P x \wedge \exists x \neg P x$. Let us compare the meaning of the universal quantifier in the upper limit logic with its meaning in the lower limit logic $\mathbf{C L}$. The former is only stronger than the latter in that less information is required for a universally quantified formula to hold true (a single instance and even the corresponding existentially quantified formula is sufficient). The upper limit meaning is typically invoked by the conditional rule, RC, which introduces a new condition in view of its defeasible character. Once the universally quantified formula is obtained, other formulas may be derived from it by the unconditional rule, RU, (carrying over the condition). However, the upper limit meaning of the universal quantifier is not different from its lower limit meaning in this respect, viz. with respect to the formulas that are derivable from a universally quantified formula. In other words, once the adaptively derivable generalizations have been added to the theory, one may 'reaxiomatize' the theory and the result may be seen as a CL-theory. ${ }^{20}$

So far for the two-logic view. The dialetheist view is radically different from it, but it is more restricted because it only concerns negation. For a dialetheist like Graham Priest, there is a 'true logic', viz. the paraconsistent LP. ${ }^{21}$ An adaptive logic that has $\mathbf{L P}$ as its lower limit logic is ampliative for the dialetheist (because $\mathbf{L P}$ is the standard of deduction). Applications of RC are justified by the so-called consistency presumption: that most inconsistencies are false and hence that they may be taken to be false unless it is found that the premises require the opposite. So the true logic alone determines the meaning of the logical terms (in a sense this is a hyperclassical position). The consistency presumption offers reasons to accept additional consequences. These, however, do not follow by logic but by logic together with the consistency presumption. Put differently, the consequence set is changed, but the meaning of negation is not.

I do not know what a dialetheist would make of the meaning of logical terms in other, for example ampliative, adaptive logics. I guess that the answer would be that deductive logic determines the meaning of the logical symbols whereas methodological steps do not affect meanings, even if they allow one to derive certain conclusions that do not follow by logic.

\footnotetext{
${ }^{19}$ I simplify here. The actual adaptive logic I have in mind here (see $\left.[15,13]\right)$ imposes certain restrictions on $A$.

${ }^{20} \mathrm{I}$ here consider the case in which no new premises are added to the theory. Indeed, if new data are gathered, these may falsify the adaptively derived generalizations and hence trivialize the so extended 'reaxiomatized' theory.

${ }^{21}$ The implication is defined in terms of the paraconsistent negation, viz. by $\neg A \vee B$, whence it is not detachable. In later versions, Priest added a modal implication to $\mathbf{L P}$, which he later replaced by a relevant implication.
} 
A more interesting question to be answered by a dialetheist concerns theories that were intended to be handled by the 'true logic', but turned out trivial. Especially if a detachable implication is around, a mathematical theory, for example, may turn out to be trivial. So suppose that the dialetheist's set theory is found to be trivial. In order to replace it by a non-trivial improvement, the dialetheist will reason from the trivial set theory. The only way to do so, as far as I see, is by considering an adaptive logic that has the 'true logic' as its upper limit and that has as lower limit a logic according to which the set theory is non-trivial. Only this approach will lead to a maximal non-trivial interpretation of the set theory. If such an approach is followed, the meaning of the logical symbols cannot be defined by the 'true logic' because this results in triviality.

If the true logic is $\mathbf{L P}$ (without a detachable implication) as was Graham Priest's view before the first edition of [21], the problem can be neglected because $B_{1} \wedge$ $\ldots \wedge B_{m}(m \geq 1)$ is a $\mathbf{L P}$-consequence of $\left\{A_{1}, \ldots, A_{n}\right\}$ iff every $B_{i}(1 \leq i \leq m)$ is a LP-consequence of a single $A_{j}(1 \leq j \leq n)$. So a theory is only trivial iff, for every formula $A$, there is an axiom $B$ of the theory from which $A$ is $\mathbf{L P}$-derivable. The presence of a relevant implication, however, changes the matter drastically.

Finally, let us turn to the direct view. According to this, the adaptive logic itself determines the meaning of logical symbols. So meaning is explicitly contextual. Let me explain in which sense this view is different from the two-logic view.

First a technical matter. Some adaptive logics, for example the logic of inductive generalization LI, have the trivial logic $\mathbf{T r}$ as their upper limit. ${ }^{22}$ To say that a logical term receives the meaning assigned to it by $\mathbf{T r}$ is a bit of a nonsense. So it seems more sensible that $\mathbf{L I}$ allows one to derive a universally quantified statement $\forall A$ from one or more instances of it, unless and until a statement falsifying $\forall A$ (for example, $\exists \neg A$ ) is derived from the premises. ${ }^{23}$ This only affects the universal quantifier and, as long as the line on which the universally quantified formula is derived is unmarked (viz. as long as no formula falsifying $\forall A$ is derived), the change to the meaning of the universal quantifier reduces to the fact that the formula can be introduced on the basis of an instance.

The relevant philosophical question, which is much more general, is whether deductive logic can be separated from defeasible logic. Technically this separation is obviously possible. The question, however, is meant in the epistemological sense. Is it possible, within a given problem solving context in which reasoning occurs, to construct theories about the meaning of logical terms in such a way that these theories are independent from the meanings of the non-logical terms? The standard format of adaptive logics ${ }^{24}$ was devised in such a way that this separation is maintained. It was not shown, however, that all defeasible reasoning will eventually be

\footnotetext{
${ }^{22}$ Do not confuse $\operatorname{Tr}$ with the modal logic Triv. Tr is characterized by $\Gamma \vdash \operatorname{Tr} A$ for all $\Gamma$ and $A$. It either has no models or only one, viz. the trivial model.

${ }^{23}$ This is not fully accurate. A disjunction of statements of the form $\exists \neg A$ will have the same effect in view of the marking definition. This is one of the reasons why the LI-consequence set of a consistent premise set is always consistent.

${ }^{24}$ A first attempt to formulate it was made in [7]. The present version is in [12] and especially in [14, Chap. 4].
} 
integrated in the present standard format of adaptive logics or in a format that allows for the separation. In other words, it is possible that our theories about the world fix the meaning of logical terms in a such way that no separate theory about the logical terms can be split off. This might especially be the case in 'provisional' stages of those theories, in which lots of theoretical problems are still to be solved by means of defeasible reasoning forms ${ }^{25}$ If such a situation obtains, one may still devise deductive logics but their application will be restricted if not empty and their use spurious.

Needless to say, the meaning of the logical symbols is determined by the derivability relation, not by what is actually derived (at a stage or finally) in a proof from a premise set. This is not any different from deductive logics. For example, that $p$ was not derived from $p \wedge q$ in a given proof does nor affect the meaning of (this) conjunction.

\section{Complex Theories}

Until now, I considered adaptive logics as methods, so as mainly relevant for the development of theories and for their application. But might such logics not also be employed as underlying logics of theories? I shall offer a brief argument to show that this is meaningful and, especially in view of Gödel's incompleteness theorems, offers interesting perspectives.

A theory is often seen as a couple comprising a decidable set of axioms and a logic, $T=\langle\Gamma, \mathbf{L}\rangle$. The set of theorems of the theory, with which the theory is sometimes identified, is taken to be $C n_{\mathbf{L}}(\Gamma)$.

Up to the nineteenth century, the logic of most theories was implicit and only rarely were the axioms listed. Yet theories were considered as well-defined and apparently also as effectively decidable (although the concept itself was not explicitly around). In the early twentieth century, it was discovered that CL is only semirecursive. So (predicative) theories, even if well-defined, are only semi-recursive consequence sets of a recursive set of axioms. At the same time, Gödel's incompleteness theorems revealed grave restrictions-see Sect. 2. Theories with other underlying logics were proposed. These were either also semi-recursive, for example when the logic was intuitionistic or relevant, or else they were much more complex, for example when the underlying logic was second order-the reader will remember that second-order logic requires infinitary rules.

By an adaptive theory I obviously mean a theory $T=\langle\Gamma, \mathbf{L}\rangle$ in which $\mathbf{L}$ is an adaptive logic. For such theories $C n_{\mathbf{L}}(\Gamma)$ is not in general semi-recursive-it may be up to $\Pi_{1}^{1}$-complex-see [25].

The reasons for introducing adaptive theories is that the world may be so complex that it cannot be captured by semi-recursive theories. Or rather, we know that the world is so complex in view of Gödel's first incompleteness theorem. If it is captured

${ }^{25}$ Some defeasible reasoning forms only concern application problems. Abduction is a ready example. 
by an adaptive theory, this theory does not have certain nice properties of traditional first-order theories, but no nice theory can capture the domain anyway. Moreover, adaptive theories have certain relatively nice properties. For one thing, they define theories (in the sense of sets of theorems) just as second-order logics. Their proofs at a stage are simple in that they proceed in terms of finitary rules; in this sense they are much simpler than second-order theories. These proofs explicate actual reasoning and introduce a kind of control, in terms of the conditions and the Marking definition, that is absent in actual reasoning. Finite adaptive proofs-at-a-stage are not more complex than, for example, CL-proofs. Even infinite adaptive proofs are relatively simple, given that they consist of a denumerable set of lines and that all applied rules are finitary (every conclusion is drawn from finitely many formulas preceding it). Heuristic procedures for adaptive proofs are available. Moreover, there are procedural criteria for final derivability (see [10] and especially [26]), whence certainty can be gained about at least a number of theorems of the theories. Note that, where a criterion applies, it establishes final derivability in a finite proof.

These criteria are worth a further comment. Establishing final derivability requires in principle that one offers an argument about all (finite or infinite) extensions of a finite proof. There are clearly more than countably many such extensions. Establishing final derivability in terms of a procedure reduces the complexity of the required reasoning. It is sufficient to establish that a finite set of formulas cannot be derived from the (decidable) premise set. The premise set is always countable and non-derivability is established in terms of the 'positive part' relation, which is decidable.

Even when no criterion applies, proofs at a stage give us the best estimate of the theory that can be obtained in view of present insights, which are the insights provided by the present proofs. This is a basis for drawing a defeasible conclusion.

I have some results that relate to Gödel's second incompleteness theorem: how to deal with a possibly inconsistent axiom system for arithmetic? As I shall write up these results soon, I do not mention them here. Moreover, the reader will be more interested in the question whether adaptive theories may be complete with respect such domains as true arithmetic (the formulas verified by standard model). Right now, Peter Verdée has ideas on the matter that look extremely promising to me-I advise the reader to look out for forthcoming results. For now, let me restrict myself to a promise. If true arithmetic is consistent, it is likely that an adaptive theory is complete with respect to it and that every formula which one can show to be verified by the standard model, for example the Gödel sentence, can be shown to be a theorem of the adaptive theory. If true arithmetic is inconsistent, the standard model (and most of model theory) is nonsense. So the classicist looses everything she has. In that case, it is extremely likely that there is an inconsistency-adaptive theory which is non-trivial and actually behaves for all natural numbers (the numbers of the standard block) as true arithmetic was intended to behave. 


\section{A Form of Logical Pluralism}

In Sect. 2, I claimed that all meaning is contextual. In this section, I offer some further arguments for that claim. These arguments go along with my epistemological stand, but may be considered independently of it. Let me admit at once that this section is somewhat touchy. Some papers opposing logical pluralism dragged me into the scene, but I felt deeply misunderstood, accused of things I never stated, associated with positions I consider utterly mistaken. If one is misunderstood, one may attack the 'opponent'. One may also feel guilty. After all, if X writes out Y's position, $\mathrm{X}$ will construct $\mathrm{Y}$ 's view on the basis of X's view. Who is to blame for misunderstanding? So let me try to be constructive and make another attempt to state my position. Part of the statement is determined by certain misunderstandings, but I shall not bother the reader with them.

The aim of logic is to explicate reasoning. What is 'out there' is actual reasoning and it has to be explicated. It is not a matter of fact. It is not a platonic heaven. It is not a domain that has to be described. So no descriptive theory of actual reasoning will do. The explicandum contains mistakes and there is a normative dimension.

As our culture likes distinctions, let us make them. Some of the reasoning is deductive, some is defeasible. Deductive reasoning can be separated into formal reasoning and informal reasoning. ${ }^{26}$

Formal deductive reasoning concerns logical terms. Its correctness is judged in view of the meanings of logical terms. This is the reason why deductive (formal) logics are supposed to fix the meanings of logical terms.

Among the logical terms that extremely frequently occur in actual reasoning are causal relations, time and tense, deontic operators, and sundry kinds of other modalities. All these are neglected by $\mathbf{C L}$ and actually by most other Tarski logics. More importantly, there is obviously a manifold of each of these. Just think, for example, of logical modalities, nomological (or 'physical') modalities, practical modalities (it is physically possible but practically impossible to bring the moon into a different orbit tomorrow), and so on. In order to make their case, monologists should articulate a single logical system that deals with all logical terms, a matter far from realized today. Moreover, they should be able to use their logical system to axiomatize all required mathematical theories as well as all empirical theories.

Some monologists will argue that there is no objection against axiomatizing a mathematical theory by means of another logic $\mathbf{L}$, as a merely technical realization as it were. The idea is that the theorems of the so obtained theory are then combined with other, for example empirical, statements in order to forge empirical theories. Note that this will only do if $\mathbf{L}$ is at least as strong as the true logic. In other words, the true logic should be conservative with respect to every mathematical theory. If it is not, non-theorems of the mathematical theory (and of its language) will

\footnotetext{
${ }^{26}$ Defeasible reasoning can also be separated into formal reasoning and informal reasoning. Adaptive logics, for example, characterize formal defeasible reasoning forms. However, I shall not need this distinction in the sequel.
} 
be derivable by the true logic and hence will ruin the applicability of the original mathematical theory.

For the sequel of this section, let us restrict attention to the traditional logical terms, say those of the predicative language schema. I shall argue that even with this restriction there are reasons for logical pluralism.

Why should the traditional logical terms be unique? Why should only one negation, one implication, one universal quantifier, ... occur in reasoning? Everyday practice clearly points to the opposite. Some negations are paraconsistent while others clearly are not. Some implications are contrapositive or transitive, while others clearly are not—see also below, where I come to the distinction between formalization and logical inference, but daily practice clearly favours a multiplicity of logical terms. So the burden of proof is on those that argue for uniqueness and, claims apart, they did not produce any sound arguments.

Once we grant that there is a multiplicity of unambiguous logical terms, why should all unambiguous logical terms occur in all contexts? Whether "context" is understood here as linguistic context or as problem-solving situation, the facts plea in favour of a negative answer to the question. So the burden of the proof is again with those that favour a positive answer. Again, prejudice apart, they failed to produce any sound arguments.

Once we grant that not all unambiguous logical terms occur in all contexts, why should a unique logic $\mathbf{L}$ be a suitable explication of the logical terms that occur in all contexts? Let $\mathbf{L}$ be a suitable explication for the logical terms that occur in a context in which we reason about beers. Why should $\mathbf{L}$ also be a suitable explication of the logical terms that occur in the context in which we reason about $\mathbf{L}$ ? The burden of the proof...

Let me interrupt this for a moment. Many classical logicians, relevance logicians, dialetheists, ... just take it for granted that there is a 'true logic' $\mathbf{L}$ and that $\mathbf{L}$ should be the logic of the metatheory of $\mathbf{L}$. I tried to stepwise spell out what they take for granted in order to arrive at this conclusion. I stepwise asked for arguments. All I got, looking at the literature, is the well-known "it is obvious that" (sometimes phrased as "it is reasonable to take it that"). But let me go on to the final step on deductive logic.

As soon as we grant that a logic may differ from the logic of its metatheory, the following seems justified. There is no 'true logic' of which parts are used in a 'context'. In other words, there is no 'true logic' that comprises the logical terms used in all possible contexts $\left(\neg_{1}, \neg_{2}, \ldots, \supset_{1}, \supset_{2}, \ldots, \wedge_{1}, \wedge_{2}, \ldots, \ldots\right)$ and from which the right logical terms are chosen according to the context. While the burden of the proof is still on those who claim there is such a logic, let me add some arguments to show that the burden is heavy. (i) Joining logics may ruin the meaning of the involved logical terms and, worse, may have tonk-like effects (cause the joined logic to be identical to the aforementioned trivial logic Tr). (ii) The 'true logic' cannot itself determine the contextual choice of terms. (iii) Two unambiguous logical terms may be equally suitable explications for the same bit of reasoning. Note that (ii) and (iii) are the reason why monologists candidly separate the formalization of natural language arguments from logical inference and, equally candidly, leave the 
formalization part unexplicated. Proceeding thus, they put themselves into a quite comfortable position. Formalizing your statements by means only known to God, they then decide what follows from your formalized statements according to their 'true logic'. In this way, they move the burden of the proof to you. If you drew a conclusion they reject, you have to find, in their logical system, a formalization of your statements from which follows your conclusion. Similarly if you reject a conclusion they draw.

From here, we move on quickly. Let us first move to informal deductive reasoning. This concerns reasoning that is correct in view of the meanings of non-logical (or referring) terms. To these applies all that was said about logical terms, but there is more. Referring terms are vague, ambiguous, etc. If in doubt, open a dictionary. Referring terms are also theory-laden. It does not follow that they are incommensurable (theory-ladenness need not prevent communication).

Finally turning to defeasible reasoning, note that all that was said before (about logical and referring terms) applies here as well. Even more than for deductive reasoning, defeasible reasoning requires an explication. The idea that anything a priori would be involved is as crazy as outdated-see the quotation from Dudley Shapere.

The first line of argument started from human reasoning. Let me briefly follow a second line of argument. Many people take it that knowledge about logical terms should not be obtained by starting from actual reasoning. They hold that there are other ways for obtaining such knowledge and that these refer to a more objective basis for logical terms.

For a start, let it be mentioned that logical terms are not hardwired in our brains. In a sense, classical logic is hardwired in digital computers, but that does not prevent one from writing programs for implementing other logical terms. While our knowledge about the functioning of human brains is far from perfect, we have reasons to believe that human brains are very different from digital computers. Our present knowledge about brains does not reveal any hardwired logical terms and rather supports the claim that logical terms are neurologically complex entities. Moreover, we are able to handle a variety of logics (classical, intuitionistic, relevant, ...) and nothing suggests that one of these is the basis from which the other logical terms can be implemented in our brains.

It is sometimes claimed that truth-preservation provides access to the true meanings of logical terms. This seems putting the cart before the horse. Indeed, in order to find out which inferences are truth-preserving, one needs to know the truth conditions of the logical terms. Thus, if $\supset$ is the implication of $\mathbf{C L}$ or of intuitionistic logic, then the inference from $A$ to $B \supset A$ is truth-preserving; if the implication is relevant, the inference is not truth-preserving. A (coherent) semantics fixes those truth conditions and hence fixes which inferences are sound. A (coherent) formal system also fixes those truth conditions because it determines an inferential semantics. This is usually described in terms of a set of two-valued valuation functions $v$. The transition from the formal system to the semantics is obtained by translating every correct inferential statement $A_{1}, \ldots, A_{n} \vdash B$ to: "for all $v, v\left(A_{1}\right)=0$ or $\ldots$ or 
$v\left(A_{n}\right)=0$ or $v(B)=1 "{ }^{27}$ The conclusion of all this is that a logic fixes (its own) truth-preservation and hence that truth-preservation cannot be used as a criterion for finding 'the true logic'. ${ }^{28}$

Others claim that conceptual analysis provides access to the true meanings of logical terms. The criticism to this view is all in line with the one in the previous paragraph. The central question is which concepts are analysed. Intuitionistic disjunction is clearly different from classical or relevant disjunction, but both seem equally coherent. Similarly for implication: the classical, intuitionist, and relevant concepts are different (and relevant implication has many variants). This is not only typical for logical terms but also for mathematical ones. Cantor's and Frege's set theories were shown trivial and hence incoherent. Possibly consistent replacements that have $\mathbf{C L}$ as their underlying logic are $\mathbf{Z F}$, NF, and several others. Each of these clearly concerns (or rather introduces) a different concept of set membership, and hence a different set concept. A nice example in this realm is Zach Weber's set theory from [27]. Here the concept of set inclusion is intensional. Where $x$ and $y$ are sets, $x=y$ iff the condition for being a member of $x$ is equivalent to the condition for being a member of $y$, where equivalence is relevant. It follows that there are infinitely many sets that, for example, have no member, but are not identical to each other-and similarly for other sets. This clearly is not in line with the tradition that locates set theory in the domain of extensionality, and it restricts the possible application contexts. Nevertheless, the underlying concept seems quite coherent and well-analysed.

Returning to the main point, it is possible that, in the end, only one concept of each kind (one negation, one implication, etc.) would turn out to be coherenttogether they would form 'the true logic'. This does not seem very likely, however. That there are different notions of coherence makes it even less likely. The stronger form of coherence one adopts, the less likely is the warrant for uniqueness. If coherent is taken to mean non-trivial, then uniqueness becomes very unlikely. Indeed, it would mean that at most one logic known today would be non-trivial. ${ }^{29}$ Moreover, the non-trivial logical concepts would have to be such that they tolerate no weakening - every weakening of the 'unique' logical terms is bound to warrant nontriviality-see next paragraph. If coherent is meant as stronger than non-trivial, I am not sure that incoherence is very fatal. Nearly all creative episodes, in empirical and logico-mathematical sciences alike, were incoherent according to some notions of coherence.

There is a limit to weakening logical terms. Consider a logical term that has no meaning at all. So it may be deleted from every string of symbols in which it occurs, without the meaning of the string being changed. Such a symbol clearly serves no

\footnotetext{
${ }^{27}$ Such an inferential semantics is often 'ugly' and sometimes not even recursive. Nicer results are sometimes obtained by translating the inferential statements to a worlds semantics. In [24], Suszko has shown that every 'logic' has a two-valued semantics; in [22], Routley and Meyer have shown the same for a two-valued worlds semantics.

28 This holds even for logics phrased in natural language, like Aristotle's syllogistics. The syllogistics determines to which occurrences of "all", "some", etc. it is legitimately applied.

${ }^{29}$ A $\operatorname{logic} \mathbf{L}$ is trivial iff $\Gamma \vdash_{\mathbf{L}} A$ for all $\Gamma$ and $A$.
} 
purpose and does not contribute anything to logic. Phrased differently, empty logic, viz. the logic $\mathbf{L}$ according to which $\Gamma \nvdash_{\mathbf{L}} A$ for all $\Gamma$ and $A$, is coherent but does not explicate or enable any reasoning. Note, however, that another possibility reveals itself at this point. We have seen in Sect. 5 that there is an adaptive logic based on zero logic. Applied to a consistent set of premises, this adaptive logic delivers exactly the same consequence set as classical logic. Applied to an inconsistent set of premises, the adaptive logic will interpret the premise set as normally as possible-read this as: as much as possible in agreement with $\mathbf{C L}$ (or with whatever the upper limit logic is chosen to be). So the lower limit logic assigns no meaning to logical terms, but the adaptive logic interprets them as much as possible in agreement with $\mathbf{C L}$ (or with whatever the upper limit logic is chosen to be). This means that the meaning of all logical terms becomes context-dependent, viz. depends on the contents of the premises.

Incidentally, I consider brain science, truth-preservation and conceptual analysis as important instruments (of which the first is beyond logicians' competence). I only argued that they are not sound means to arrive at 'the true logic'.

After having attacked means invoked by monologists, I now turn to means that I would consider conclusive. Actually, I see only one. We (humans) are striving for obtaining a body of useful knowledge. I write "useful" rather than "complete", because completeness seems out of reach anyway. By writing "useful", I also mean to exclude unimportant and irrelevant knowledge. Ideally, this body of knowledge should form a single theory. We are far away from that stage today. Also, we had better stick to partial and problematic theories rather than opting for a unified but weaker theory-unification is only a relative merit. Nevertheless, striving for unification is important because it reveals problems and sometimes enables us to solve them. That the adequate body of knowledge is located at the proverbial end of time, should not prevent us from striving towards it.

Note that the adequacy of a body of knowledge is a function of the world as well as of our knowledge capacities. The latter are limited. This is why the world may be so complex that we cannot consistently describe it by the means available to us, for example denumerable languages. If the inconsistent description is richer and more precise than any consistent one, then every scientist will obviously opt for the inconsistent description.

In the ideal body of knowledge, all theories (logical, mathematical, and empirical) should form a coherent structure. What will matter most are obviously the empirical theories. But these will require mathematical theories and the empirical theories, together with the mathematical ones, will require logical theories. If this would reveal that there is a unique true logic, then I shall gladly admit that there is a true logic.

The reader may have read the previous paragraph as saying that mathematics is the servant of empirical theories and that logic is the servant of both (remember that Thomas Aquinas saw philosophy as the servant_-"ancilla" he said, which is a female and not the female of "servus"- of theology). This is not what I meant. What I did mean, however, is that the world, as knowable by us, who are parts of the world, is the correct criterion. 
Does this mean that, in the end, I favour monologism? By no means. First, we are not and never shall be at the proverbial end of time. So we have no idea of what 'the true logic' is, if there is one. Next, even at the proverbial end of time, it is still possible that different theories will require different logics. Even at the proverbial end of time, some theories may require a different underlying logic than others. Coherence only supposes that, if a theory $T$ is used to formulate a theory $T^{\prime}$, then $T$ has a logic that is at least as strong as the underlying logic of $T^{\prime}{ }^{30}$

The third argument against end-of-time monologism deserves a separate paragraph. Suppose that a unique logic turns out to be revealed by the adequate body of knowledge at the end of time. So this is 'the true logic'. Yet, what use is it to us? We are not in the ideal end-of-time situation. We have to cope with the present transitory theories. Handling these may require (defeasible as well as deductive) logics that are very different from the true logic. This is an understatement. Even physics is not unified today. There never was as much disagreement on its fundamentals than in our era-just compare string theories (and their difficulties) with particle physics. That the end-of-time logic might be adequate for all contemporary theories seems (put politely) unlikely or (put bluntly) nonsensical.

I have presented two lines of argument. I hope they made the reader doubt, and hence think - all a philosopher can hope is to make his audience think. Yet, I realize very well that there is a question that should be answered by me. If there indeed is this plurality of logics, defining meanings for logical terms, in which way should we choose which logic applies in which context?

The "we" being ambiguous, let me disambiguate it. Non-logicians make the choice intuitively in terms of their learned implicit reasoning competence. Whether this is better or worse is not our concern. If it is worse, we logicians have to teach them. For us logicians, the task is straightforward but not simple: we have to study properties of logics to make a justified choice possible. Needless to say, lots of work still has to be done. I realize this from personal experience. For many years I have been teaching my freshmen the logic PCR, which is the extension of propositional CL with a very simple but relevant implication. I think this logic is able to capture most of natural language reasoning, but I admit that a systematic study is lacking.

By all means, the criterion for choosing between logics is satisficing rather than optimizing. Given the obviously lacking survey of all possibilities, optimizing is simply out of reach; satisficing to the contrary is sufficient. This solves many problems. For example, it relieves us from the impracticable task to find a weighed average of the different merits of different logics.

In practice, things are rather simple; the motto is: pick a choice and look for counterarguments. Even if this leads to a choice that is 'pragmatic' and provisional, not much harm will be done provided one keeps track of what happens if things go wrong. Allow me to give a rather personal example here. I think I found a way to preserve most of Peano Arithmetic even if Peano Arithmetic is inconsistent- $\mathrm{I}$ referred to this in Sect. 7. The means are to replace Peano Arithmetic by a CLequivalent axiom system and to replace $\mathbf{C L}$ by a specific inconsistency-adaptive

${ }^{30}$ If the underlying logic of $T$ is adaptive—see Sect. 7—then the logic of $T^{\prime}$ should not be stronger that the adaptive logic's lower limit. 
logic. From a pragmatic point of view, however, I would advise a mathematician to go on using $\mathbf{C L}$ as underlying logic (and hence not to keep track of the conditions that the inconsistency-adaptive logic requires). I know this will sound outrageous to my paraconsistent friends. And yet, I have a good reason for this advice. As long as no inconsistency is derived from Peano Arithmetic, CL will enable us to derive theorems of Peano Arithmetic that would also be derivable at-a-stage by the inconsistency-adaptive logic — never mind what this logic precisely is. Once an inconsistency is (some would like to say "were") derived from the Peano axioms, there is an algorithm for transforming every CL-proof into the inconsistency-adaptive proof and for deciding which lines of the inconsistency-adaptive proof are marked. So writing CL-proofs is harmless, provided we realize that an inconsistency may turn up and that we know what we have to do in that case.

Acknowledgements Research for this paper was supported by subventions from the Fund for Scientific Research - Flanders and from Ghent University. I am indebted to Peter Verdée and to the two referees for careful comments on previous drafts.

\section{References}

1. Batens, D.: Incommensurability is not a threat to the rationality of science or to the antidogmatic tradition. Philosophica 32, 117-132 (1983)

2. Batens, D.: Meaning, acceptance, and dialectics. In: J.C. Pitt (ed.) Change and Progress in Modern Science, pp. 333-360. Reidel, Dordrecht (1985)

3. Batens, D.: Do we need a hierarchical model of science? In: J. Earman (ed.) Inference, Explanation, and Other Frustrations. Essays in the Philosophy of Science, pp. 199-215. University of California Press (1992)

4. Batens, D.: Menselijke kennis. Pleidooi voor een bruikbare rationaliteit. Garant, Antwerpen/Apeldoorn (1992). Second edition: 2004; third edition: 2008.

5. Batens, D.: On the epistemological justification of pluralism and tolerance. In: Philosophie et Tolérance. Philosophy and Tolerance. Actes des Entretiens de Rabat, vol. I, pp. 33-54 (2000). [= Philosophica 65.$]$ Appeared 2002

6. Batens, D.: A survey of inconsistency-adaptive logics. In: D. Batens, C. Mortensen, G. Priest, J.P. Van Bendegem (eds.) Frontiers of Paraconsistent Logic, pp. 49-73. Research Studies Press, Baldock, UK (2000)

7. Batens, D.: A general characterization of adaptive logics. Logique et Analyse 173-175, 45-68 (2001). Appeared 2003

8. Batens, D.: A formal approach to problem solving. In: C. Delrieux, J. Legris (eds.) Computer Modeling of Scientific Reasoning, pp. 15-26. Universidad Nacional del Sur, Bahia Blanca, Argentina (2003)

9. Batens, D.: The need for adaptive logics in epistemology. In: Logic, Epistemology and the Unity of Science, pp. 459-485. Kluwer Academic Publishers, Dordrecht (2004)

10. Batens, D.: A procedural criterion for final derivability in inconsistency-adaptive logics. Journal of Applied Logic 3, 221-250 (2005)

11. Batens, D.: Content guidance in formal problem solving processes. In: O. Pombo, A. Gerner (eds.) Abduction and the Process of Scientific Discovery, pp. 121-156. Centro de Filosofia das Ciências da Universidade de Lisboa, Lisboa (2007)

12. Batens, D.: A universal logic approach to adaptive logics. Logica Universalis 1, 221-242 (2007) 
13. Batens, D.: Logics for qualitative inductive generalization. Studia Logica 97(1), 61-80 (2011)

14. Batens, D.: Adaptive Logics and Dynamic Proofs. Mastering the Dynamics of Reasoning (201x). Forthcoming

15. Batens, D., Haesaert, L.: On classical adaptive logics of induction. Logique et Analyse 173175, 255-290 (2001). Appeared 2003

16. Batens, D., Provijn, D.: Pushing the search paths in the proofs. A study in proof heuristics. Logique et Analyse 173-175, 113-134 (2001). Appeared 2003

17. Heeffer, A.: Abduction as a strategy for concept formation in mathematics: Cardano postulating a negative. In: O. Pombo, A. Gerner (eds.) Abduction and the Process of Scientific Discovery, pp. 179-194. Centro de Filosofia das Ciências da Universidade de Lisboa, Lisboa (2007)

18. Heeffer, A.: The symbolic model for algebra: Functions and mechanisms. In: L. Magnani, W. Carnielli, C. Pizzi (eds.) Model-Based Reasoning in Science and Technology. Abduction, Logic, and Computational Discovery, Studies in Computational Intelligence, vol. 314, pp. 519-532. Springer, Heidelberg (2010)

19. Hintikka, J.: Inquiry as Inquiry: A Logic of Scientific Discovery. Kluwer, Dordrecht (1999)

20. Meheus, J., Batens, D.: Steering problem solving between cliff incoherence and cliff solitude. Philosophica 58, 153-187 (1996). Appeared 1998

21. Priest, G.: In Contradiction. A Study of the Transconsistent. Oxford University Press, Oxford (2006). Second expanded edition (first edition 1987)

22. Routley, R., Meyer, R.K.: Every sentential logic has a two-valued worlds semantics. Logique et Analyse 71, 345-364 (1976)

23. Shapere, D.: Logic and the philosophical interpretation of science. In: P. Weingartner (ed.) Alternative Logics. Do sciences need them?, pp. 41-54. Springer, Berlin, Heidelberg (2004)

24. Suszko, R.: The Fregean axiom and Polish mathematical logic in the 1920s. Studia Logica 36, 377-380 (1977)

25. Verdée, P.: Adaptive logics using the minimal abnormality strategy are $\Pi_{1}^{1}$-complex. Synthese 167, 93-104 (2009)

26. Verdée, P.: A proof procedure for adaptive logics. Logic Journal of the IGPL 21, 743-766 (2013)

27. Weber, Z.: Transfinite numbers in paraconsistent set theory. Review of Symbolic Logic 3, 71-92 (2010)

28. Wiśniewski, A.: The Posing of Questions. Logical Foundations of Erotetic Inferences. Kluwer, Dordrecht (1995)

29. Wiśniewski, A.: The logic of questions as a theory of erotetic arguments. Synthese 109, 1-25 (1996)

30. Wiśniewski, A.: Erotetic search scenarios. Synthese 134, 389-427 (2003)

31. Wiśniewski, A.: Erotetic search scenarios, problem-solving, and deduction. Logique et Analyse 185-188, 139-166 (2004). Appeared 2005 